\title{
Diffusion of $\alpha$-Tocopherol in Membrane Models: Probing the Kinetics of Vitamin E Antioxidant Action by Fluorescence in Real Time
}

\author{
Gabriela Gramlich, ${ }^{\dagger}$ Juayun Zhang, ${ }^{\dagger}$ and Werner M. Nau ${ }^{*, \dagger, \star}$ \\ Contribution from the Departement Chemie, Universität Basel, Klingelbergstrasse 80, CH-4056 Basel, \\ Switzerland and the School of Engineering and Science, International University Bremen, Campus \\ Ring 1, D-28759 Bremen, Germany
}

Analysis of Fluorescence Quenching in Triton XR-100 Micelles According to a 3D Diffusion Model with Linear Regression Analysis Instead of Global Fitting. The data of fluorescence quenching of Fluorazophore-L in Triton XR-100 micelles can alternatively be fitted according to the 3D diffusion model by the indirect method of Miller et al., that was applied, e.g., to vesicles. $^{1,2}$ This analytical procedure rests on eq. S-1 as a modification of eq. 4 (see main text). $k_{\text {app }}$ and $k_{\mathrm{DO}}$ include the concentration $\left[\mathrm{Q}_{3 \mathrm{D}}\right]$ for simplicity and were obtained from individual fitting of the decay traces to provide the quencher concentration-dependent values in Table S-1.

$$
\begin{gathered}
I_{(t)}=I_{(0)} \exp \left[-\left(k_{a p p} t+k_{D O} \sqrt{t}\right)\right] \\
\text { with } k_{a p p}=k_{0}+\mathrm{k}_{\mathrm{V}}\left[\mathrm{Q}_{3 \mathrm{D}}\right] \text { and } k_{D 0}=k_{D}\left[\mathrm{Q}_{3 \mathrm{D}}\right]
\end{gathered}
$$

$k_{\mathrm{V}}$ and $k_{\mathrm{D}}$ can be obtained in a second step from linearized regression plots of $k_{\text {app }}$ and $k_{\mathrm{DO}} v s\left[\mathrm{Q}_{3 \mathrm{D}}\right]$ as shown in Figure $\mathrm{S}-1$. The diffusion coefficient $D$ and the reaction encounter distance $R$ can then be extracted from the experimental values of $k_{\mathrm{V}}$ and $k_{\mathrm{D}}$ by using the relations

$$
R=\left(\frac{k_{D}{ }^{2}}{16 \times N_{a} \times k_{V}}\right)^{\frac{1}{3}} \text { and } D=\frac{1}{\pi}\left(\frac{k_{V}{ }^{2}}{2 \times N_{a} \times k_{D}}\right)^{\frac{2}{3}}
$$

This analysis yields a diffusion coefficient $D$ of $9.8( \pm 4.1) \times 10^{-8} \mathrm{~cm}^{2} \mathrm{~s}^{-1}$ and a radius $R$ of 11.0 $\pm 3.6 \AA$. Compared to the direct global fitting routine (see main text), the values from the linear regression method have a very large uncertainty due to error propagation in the multi-step data analysis. The method by Miller et al. is lucid due to the linearized data representation, but introduces a large error. 
Table S-1. Kinetic parameters for fitting of the fluorescence quenching of Fluorazophore-L (1 mM) by $\alpha$-tocopherol in Triton XR-100 (27 mM) micelles according to eq. S-1 for 3D viscous diffusion.

\begin{tabular}{ccccc}
\hline $\begin{array}{c}{[\mathrm{Q}] / \mathrm{mM}} \\
\text { in bulk }\end{array}$ & $\begin{array}{c}{\left[\mathrm{Q}_{3 \mathrm{D}}\right] / \mathrm{mM}} \\
\text { in surfactant }\end{array}$ & $I_{0}($ counts max $) / 10^{4}$ & $k_{\mathrm{app}} /\left(10^{7} \mathrm{~s}^{-1}\right)$ & $k_{\mathrm{D} 0} /\left(10^{3} \mathrm{~s}^{-1 / 2}\right)$ \\
\hline 0 & 0 & 1.11 & 1.81 & -- \\
0.27 & 16 & 1.11 & 1.92 & 0.53 \\
0.53 & 32 & 1.12 & 2.01 & 1.12 \\
0.80 & 48 & 1.11 & 2.13 & 2.65 \\
0.11 & 65 & 1.10 & 2.35 & 2.69 \\
\hline
\end{tabular}

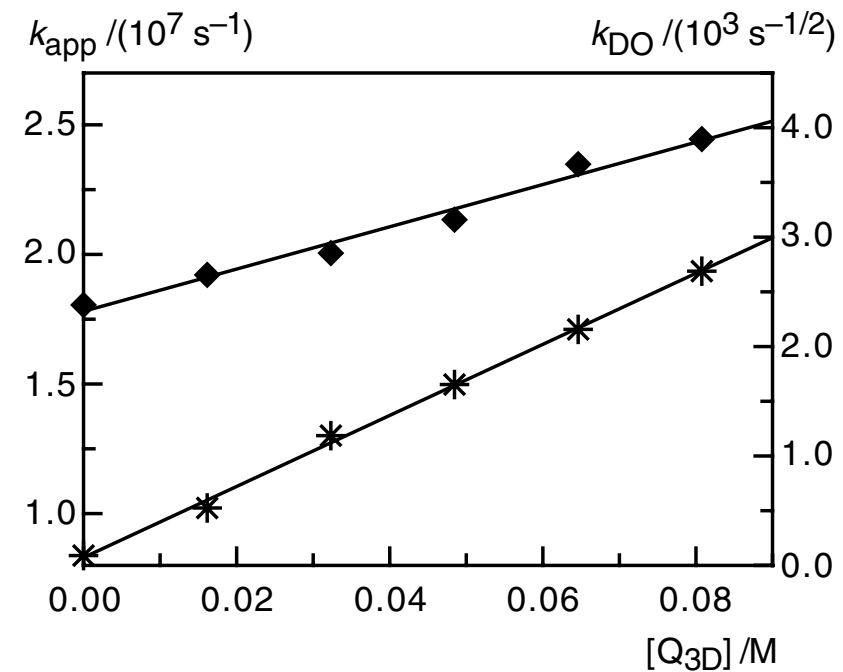

Figure S-1. Plot of $k_{\text {app }}$ (diamonds, $n=6, r=0.991$ ) and $k_{\mathrm{DO}}$ (asterisks, $n=6, r=0.999$ ) versus $\left[\mathrm{Q}_{3 \mathrm{D}}\right]$ according to the method by Miller et al. for the fluorescence quenching of Fluorazophore-L (1 mM) by $\alpha$-Toc in Triton XR-100 (27 mM) micelles. 
Stern-Volmer Treatment. The square-root terms required for an accurate analysis of the fluorescence decays in Triton micelles (diffusion in viscous solution, eq. 4 of main text) or in POPC liposomes (diffusion in 2D, eq. 3 of main text) dominate at short decay times. Consequently, all fluorescence decays in these lipidic structures become approximately monoexponential on a longer time scale. In practice, this turned out to be the case already after 40 ns, i.e., when monoexponential fits were performed by ignoring the fast decay region (the transient effect) within the first $40 \mathrm{~ns}$, one obtained reduced chi square values $<1.25$. This happenstance allows one to analyze crudely, regardless of further assumptions about dimension, size, and viscosity of the lipidic system, the fluorescence quenching in Triton micelles as well as in POPC liposomes according to a simple Stern-Volmer quenching kinetics: $I(t)=I_{0} \exp \left[-k_{a p p} t\right]$ with $k_{a p p}=k_{0}+k_{q}\left[\mathrm{Q}_{3 \mathrm{D}}\right]$. The data can be compared with the result in non-viscous solution, for which Stern-Volmer behavior is well-established.

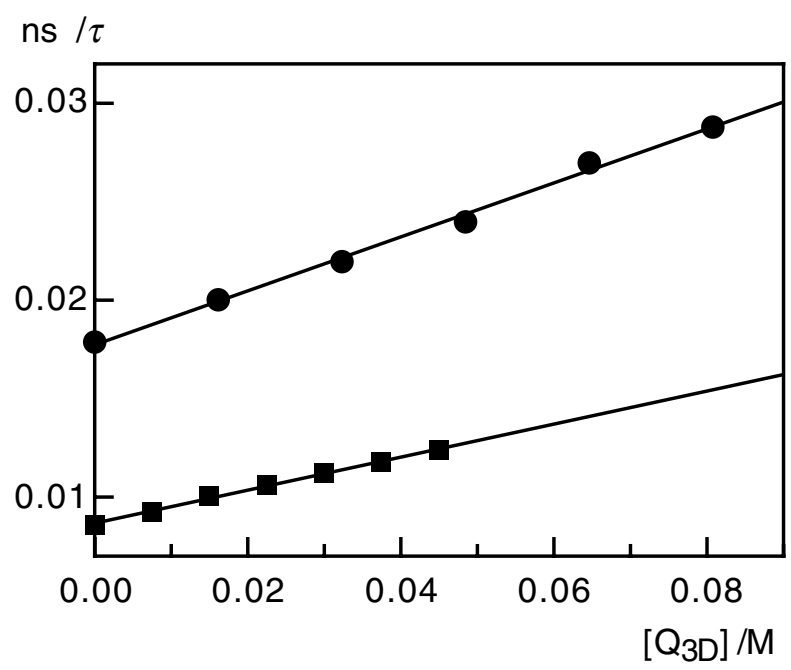

Figure S-2. Stern-Volmer plots for quenching of Fluorazophore-L by $\alpha$-Toc in $27 \mathrm{mM}$ Triton XR-100 micelles (circles, $n=6, r=0.998$ ) and $0.7 \mathrm{mM}$ POPC liposomes (rectangles, $n=7, r=0.999$ ).

Linear regression of the Stern-Volmer plots $\left(1 / \tau=k_{\text {app }}\right.$ versus $\left[\mathrm{Q}_{3 \mathrm{D}}\right]$, Figure $\left.\mathrm{S}-2\right)$ provided the bimolecular quenching rate constants, $k_{\mathrm{q}}$. Values of $1.4 \times 10^{8} \mathrm{M}^{-1} \mathrm{~s}^{-1}$ for Triton micelles and $8.4 \times 10^{7}$ $\mathrm{M}^{-1} \mathrm{~s}^{-1}$ for POPC liposomes were obtained, more than one order of magnitude slower than the quenching rate constant of Fluorazophore-L by $\alpha$-Toc in benzene $\left(3.9 \times 10^{9} \mathrm{M}^{-1} \mathrm{~s}^{-1}\right){ }^{3}$ The unimolecular quenching in SDS, which provides the quenching rate constant for a single quencher in a micelle with approximately 63 SDS molecules, can also be roughly converted to a bimolecular rate constant by conversion to a three-dimensional quencher concentration in SDS micelles. One obtains a value of 3.8 $\times 10^{8} \mathrm{M}^{-1} \mathrm{~s}^{-1}$, in good agreement with a previous estimate for a different probe/quencher system $\left(4 \times 10^{8}\right.$ $\left.\mathrm{M}^{-1} \mathrm{~s}^{-1}\right){ }^{4}$ All Stern-Volmer rate constants, as well as the estimated diffusion coefficients, are entered in Table 1 of the main text. 
Monoexponential Approximation for Lateral Diffusion. The direct monoexponential fitting of the decay traces in POPC assemblies (without the initial 40-ns region), where 2D diffusion is expected to operate, can also be employed to obtain a "two dimensional" quenching rate constant $\left(k_{\text {app }}=\right.$ $7.7 \times 10^{-7} \mathrm{~cm}^{2} \mathrm{~s}^{-1}$ ), which can be used to estimate the lateral diffusion coefficient by much simpler approximate formulas without the square-root term. This procedure (i) by-passes the necessity of complex fitting procedures, (ii) can make use of experimental decay data with poorer time resolution and (iii) does not require the reaction radius $R$ for fitting. The simple Sackmann relation $\left(D_{\mathrm{L}} \approx k_{\text {app }} / 4\right)^{5}$ affords $D_{\mathrm{L}}=1.9( \pm 0.2) \times 10^{-7} \mathrm{~cm}^{2} \mathrm{~s}^{-1}$, which is fortuitously the same as that obtained from the global fitting according to the full functional expression (eq. 3). In contrast, the equations derived from the $2 \mathrm{D}$ continuum model by neglecting the transient term in the Razi Naqvi formula in eq. $3\left(D_{\mathrm{L}} \approx k_{\text {app }} / 2.31\right)^{6}$ yield a $D_{\mathrm{L}}$ estimate of $3.3( \pm 0.2) \times 10^{-7} \mathrm{~cm}^{2} \mathrm{~s}^{-1}$ which is clearly too large. Care has to be taken in

employing such approximate treatments, ${ }^{7}$ which ignore the inherent time dependence of 2D quenching rates. ${ }^{8}$ Global fitting of the decay traces and use of the full formula by Razi Naqvi et al. ${ }^{6}$ (eq. 3) is therefore always advised as the method of choice, keeping in mind that the parameter set is optimized for a 100-ns lifetime probe.

\section{References and Footnotes:}
$\dagger \quad$ Universität Basel
$¥ \quad$ International University Bremen
(1) Miller, D. D.; Evans, D. F. J. Phys. Chem. 1989, 93, 323-333.
(2) Miller, D. D.; Magid, L. J.; Evans, D. F. J. Phys. Chem. 1990, 94, 5921-5930.
(3) Gramlich, G.; Zhang, J.; Winterhalter, M.; Nau, W. M. Chem. Phys. Lipids 2001, 113, 1-9.
(4) Almgren, M.; Löfroth, J.-E. J. Colloid Interface Sci. 1981, 81, 486-499.
(5) Galla, H.-J.; Sackmann, E. Biochim. Biophys. Acta 1974, 339, 103-115.
(6) Razi Naqvi, K.; Martins, J.; Melo, E. J. Phys. Chem. B 2000, 104, 12035-12038.
(7) Kano, K.; Kawazumi, H.; Ogawa, T.; Sunamoto, J. J. Phys. Chem. 1981, 85, 2204-2209.
(8) Caruso, F.; Grieser, F.; Murphy, A.; Thistlethwaite, P.; Urquhart, R.; Almgren, M.; Wistus, E. J. Am. Chem. Soc. 1991, 113, 4838-4843. 